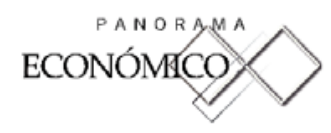

Volumen VII, No. 13

Julio-Diciembre, 2011, pp. 53-74

\title{
¿CÓMO AFECTÓ LA CRISIS ECONÓMICA GLOBAL AL SECTOR EDIFICADOR EN COLOMBIA? UN ANÁLISIS DE LOS CANALES DE CONTAGIO
}

\author{
Jaime Rafael Ahcar Olmos* \\ Lya Paola Sierra Suárez** \\ José Tomás Peláez Soto***
}

(Recibido: Abril 2011 / Aprobado: Julio 2011)

\section{Resumen}

El presente artículo analiza los canales de contagio de la crisis económica internacional en el sector edificador colombiano. Así, mediante un estudio de tipo analíticodescriptivo se examina el comportamiento de los principales determinantes de la actividad edificadora nacional durante la crisis global. Igualmente, se realiza una revisión de la literatura que explica las causas y consecuencias de las crisis financieras y económicas, y sus canales de contagio, en especial aquellos relacionados con la actividad constructora. Luego de la revisión bibliográfica y el análisis de información estadística se encontró que, la crisis financiera y económica global terminó por afectar el sector de la construcción a través de los canales comercial y financiero, los

* Magíster en Comercio Internacional de la Universidad de Alicante. Correo electrónico: jahcar@javerianacali. edu.com>.

** Magíster en Economía, Pontificia Universidad Javeriana. Economista Pontificia Universidad Javeriana. Correo electrónico:<lyap@javerianacali.edu.co>.

*** Economista, Pontificia Universidad Javeriana Cali. Correo electrónico: <jtpelaez@javerianacali.edu.com>. Este artículo es resultado del proyecto de investigación titulado "Análisis de los canales de contagio de la crisis económica global en el sector de la construcción en Cali”, derivado de la línea de investigación en Globalización y Desarrollo del Grupo de Investigación IDEAS, categorizado por Colciencias, de la Pontificia Universidad Javeriana Cali. 
cuales influyeron en la reducción en el ingreso disponible de los hogares, las remesas, las tasas de interés, las variaciones en los precios de los insumos de la construcción. No obstante, las estrategias de políticas expansivas tanto fiscal como monetaria han contribuido a compensar los efectos negativos sobre el sector y contribuir a una pronta recuperación del mismo.

Palabras clave: crisis económicas y financieras, sector edificador, Colombia, canales de contagio, ingreso disponible, tasas de interés

\begin{abstract}
This article analyzes the knock-on channels of the global economic crisis in the Colombian building sector. In this way, by means of an analytical and descriptive stu$\mathrm{dy}$, the major determining factors of the national building activity during the global crisis, are examined. Likewise, a literature review that explains the causes and consequences of the financial and economic crisis, and its knock-on channels, specially those related to the building activity is carried out. The literature review and the statiscal data analysis prompted that the financial and economic global crisis winded up affecting the building sector throughout the commercial and financial channels; which affected the available income of households, cash transferences, interest rates and price variations of building material. However, the strategies of expansive policy have contributed to compensate the negative effects on the sector and to contribute to an early recovery of the mentioned sector.

Keywords: economic and financial crisis, construction sector, Colombia, contagion channels, dispensable income, interest rates
\end{abstract}

Clasificación JEL: E30, E50, G01

\title{
Introducción
}

La crisis financiera y económica global originada a mediados de 2008 se extendió por todo el mundo afectando las perspectivas de crecimiento tanto de las economías desarrolladas como de las economías en vía de desarrollo, en lo que se conoce como efecto contagio o efecto dominó. En consecuencia, el crecimiento de la economía colombiana se vio afectado por el descenso en la producción de la economía mundial. 
Según The Economist Intelligence Unit (2010) el PIB de los Estados Unidos presentó una caída de $-2.6 \%$ en 2009 , lo que contribuyó a la reducción del crecimiento de la economía nacional que no sobrepasó el $0.8 \%$ para el mismo año.

La crisis económica global terminó por afectar el comportamiento de la economía Colombiana e influyó en la evolución del sector edificador a través de diversos canales de contagio que vale la pena estudiar.

La construcción de vivienda es una actividad relevante para analizar debido a su importancia relativa como proporción del Producto Interno Bruto (PIB), su papel como vehículo de inversión, su capacidad de generación de empleos directos e indirectos, y el efecto multiplicador o de arrastre que tiene sobre la economía en su conjunto.

En Colombia, el sector edificador representó durante el tercer trimestre de 2009 el 3.2\% del PIB nacional (DANE-b, 2009). Asimismo, durante el primer trimestre de 2010 el sector en su conjunto (edificaciones y obras civiles) ocupó a 952 mil trabajadores, los cuales representaron el 5.1\% de los ocupados en el país (DANE-c, 2010). Además, en promedio, el 22\% de la formación bruta de capital o de la inversión total del país durante el período 2000-2007 correspondió a inversión en edificaciones (CAMACOL, 2009).

Asimismo, la actividad constructora está estrechamente vinculada con el comportamiento de los demás renglones de la economía por el alto grado de encadenamientos productivos, hacia atrás con el sector minero y hacia delante con el sector industrial. ${ }^{1}$ En tal sentido, la construcción ha sido tradicionalmente considerada como un sector clave para jalonar el desarrollo económico del país, pues genera empleos directos e indirectos, y tiene un efecto multiplicador en la economía.

En consideración a lo anterior, a través de una revisión bibliográfica y utilizando una metodología analítico-descriptiva, este artículo se concentra en analizar las relaciones de las crisis financieras con el sector de la construcción y en indagar sobre los canales de contagio y las consecuencias de la crisis económica global en el segmento de la construcción en Colombia, dadas la importancia y vulnerabilidad del mismo.

La revisión bibliográfica examina los autores más renombrados en el estudio de las crisis económicas y financieras, como son Krugman, Case, Kindleberger y Bernake, entre otros, y hace referencia a estudios realizados sobre el sector de la

1 La importancia de los encadenamientos productivos para el desarrollo económico fue un aporte de Hirschman (1958), quien a su vez distinguió los tipos de encadenamientos hacia atrás y hacia adelante. Trabajos sobresalientes sobre el análisis de los encadenamientos sectoriales los han desarrollado Rasmussen (1956), Hirschman (1958 y 1961), Chenery y Watanabe (1958). 
construcción a nivel nacional, como Clavijo, Junguito y Cárdenas. Adicionalmente, este trabajo se apoya en la revisión de estadísticas suministradas por fuentes como el Departamento de Estadísticas Nacionales, DANE, la Cámara de Comercio de la Construcción CAMACOL y Bloomberg, entre otros.

A lo largo del artículo se aborda, como primera medida, el tema de las crisis financieras y económicas pasando por la comprensión de sus causas y consecuencias; seguidamente, se centra en analizar la relación entre el sector de la construcción y las crisis económicas y financieras, así como la relación entre el sistema financiero y el ciclo económico y de la construcción, para luego intentar esclarecer las implicaciones de la crisis económica mundial en la economía y, específicamente, en el sector de la construcción en Colombia. Por último, se presentan las conclusiones y recomendaciones del trabajo, y una amplia revisión bibliográfica sobre los temas tratados.

\section{Crisis financieras y económicas}

La crisis financiera global desatada a mediados de 2008 es el resultado de un largo proceso de globalización financiera acaecida en un marco de desregulación extrema representada en excesos de apalancamiento, abuso de la ingeniería financiera y otras prácticas lascivas, que si bien la retroalimentaba, también engendraba el germen de su propia destrucción (Soros, 2008) y (Krugman, 2009).

En esta línea, Kaminsky y Reinhart (1999) encontraron que la liberalización financiera generalmente precede las crisis bancarias y que, a su vez, los problemas en el sector bancario típicamente preceden las crisis económicas.

Barry Eichengreen y Michael Bordo (2002) han señalado que las crisis financieras son dos veces más comunes ahora de lo que lo eran antes de 1914. Si bien la frecuencia y el poder diseminador de las crisis se ha intensificado en los últimos años, las causas de las mismas siguen siendo muy diversas tanto en la presencia o no de ciertos acontecimientos como en la secuencia en que se producen, en otras palabras, ninguna crisis es igual a otra.

No obstante, algunos economistas como Millar (1924), desde hace décadas señalan que es común encontrar ciertas causas que suelen estar presentes en muchas de ellas, y que son importantes destacar y analizar con el fin de prevenir situaciones semejantes en el futuro.

Kindleberger et al. (2001) aseveran que las crisis financieras se retroalimentan, por lo tanto no son independientes de las sucedidas en el pasado o en otros países. 
La crisis financiera americana reciente, mejor conocida como crisis subprime, se origina en un marco de exceso de liquidez en el mundo que propició un boom crediticio, acompañado por el desbordado otorgamiento de créditos hipotecarios de dudoso recaudo o subprimes.

Esta situación se agravó aún más debido a la titularización de estos títulos hipotecarios promovida por establecimientos financieros sofisticados que, buscando deshacerse del riesgo subprime, ingeniaron mecanismos muy sofisticados de fragmentación y calificación de riesgo, como el caso de los Collateralised-debt obligations (CDO). Éstos, debido a su complejidad, daban la impresión de ser bastante fiables, sin embargo resultaron menos seguros y transparentes de lo que se esperaba, ocasionando un aumento de los riesgos del sector financiero en su totalidad.

Según Rajan y Zingales (2004), una buena manera de pensar en los CDO es verlos como un mecanismo de convertir títulos hipotecarios y bonos corporativos de enormes activos líquidos, poseídos por inversionistas locales, a instrumentos financieros líquidos que se pueden vender en todo el mundo.

A la par del boom crediticio comenzó a formarse una burbuja en el sector de la finca raíz dentro de la cual los precios se incrementaban a tasas de dos dígitos año tras año. Como toda burbuja, ésta también alcanzó su punto límite y explotó a finales de 2007. A partir de entonces los precios de la finca raíz comenzaron una espiral a la baja, la cual se frena a partir de enero de 2009, como puede verse en la Gráfica 1.

El apogeo de los créditos subprime también se atribuye a la búsqueda de rentabilidad a cualquier precio por parte la banca. "La principal causa de la crisis fue el auge y la posterior caída de los préstamos titularizados -securitized lendings-, que permitió que los bancos originaran créditos para luego reempaquetarlos y venderlos en el mercado" (Ferguson, 2008, p. 64).

La caída de este innovador mercado, junto con el creciente impago de muchas hipotecas subprime, frenaron el ciclo especulativo donde se podía pedir un préstamo, comprar un bien inmueble y venderlo a un precio sustancialmente mayor que permitiera el pronto pago de la deuda y la eventual adquisición de una nueva obligación para continuar el proceso especulativo. A partir de este momento, para los especuladores resulta mucho más rentable no pagar las cuotas y entregar el bien inmueble al banco.

No obstante, en economías como la española el mercado de préstamos titularizados no se desarrolló completamente debido a que el solicitante del crédito no puede deshacerse de la obligación tan fácilmente (Reid, 2008). 


\section{Gráfica 1}

Índice de precios de la vivienda nueva en Estados Unidos

Enero de 1994 a Marzo 2010

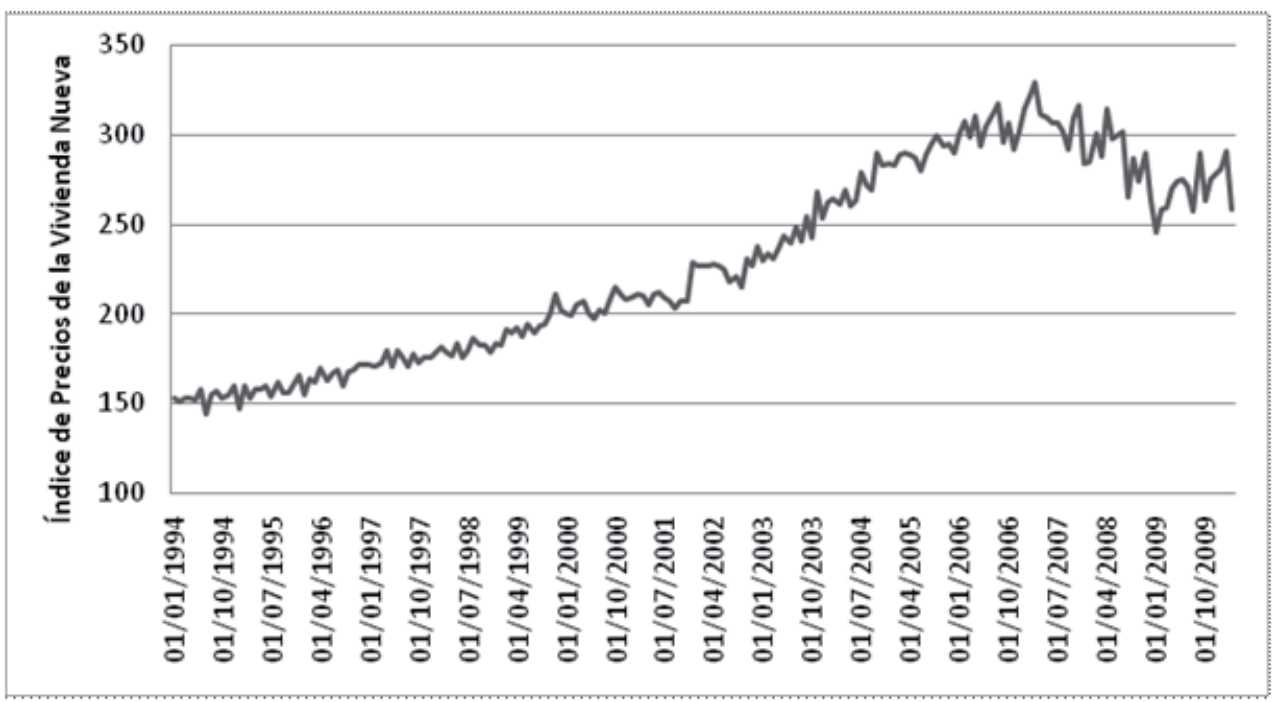

Fuente: Bloomberg. New Home Sales Index.

Posteriormente, cuando la crisis financiera se traslada al sector real, no serían solamente los especuladores quienes perderían sus hipotecas, sino muchos hogares que, tras la reducción de sus ingresos, se encontraban sin fondos para seguir pagando por gozar de un techo propio.

El crash de la burbuja inmobiliaria y su impacto en los impagos de las hipotecas, sumado a la percepción de un mayor riesgo a lo largo del sector financiero que puso en tela de juicio el riesgo subyacente de los productos titularizados, condujeron a una crisis de confianza generalizada en el sistema.

Como consecuencia de lo anterior se produjo una fractura en la concesión de créditos interbancarios que terminó por profundizar aún más los problemas de liquidez, llevando a las entidades más vulnerables a los límites que podrían soportar sus balances.

Autores como Toffler y Toffler (2008, p. 93) indican que "aunque la crisis haya sido desatada en Estados Unidos, sus efectos directos e indirectos se sentirán en todo el globo, desde Europa y China hasta India y Rusia”. 


\section{El sector de la construcción y las crisis económicas y financieras}

Forma parte de la sabiduría popular la idea de que la vivienda es una buena inversión y además una inversión segura. No obstante, este sector, al igual que el bursátil, tiene periodos de expansión y contracción que van muy de la mano con los ciclos económicos.

En este sentido, el sector de la construcción está sujeto a periodos de aumentos y disminuciones de los precios o valorizaciones de los activos. En muchas ocasiones la inversión en vivienda o activos de finca raíz no termina siendo una buena opción a largo plazo; según Shiller (2005), desde 1890 a 2004 los precios de la vivienda (descontada la inflación) se incrementaron en tan solo un $0.4 \%$ anual en los Estados Unidos. Aunque estos rendimientos mejoraron con la formación de la burbuja especulativa que incrementó los precios de la vivienda a niveles de $7.6 \%$ anual entre 2000 y 2006, la mayor parte de esa rentabilidad se ha evaporado con la brusca desvalorización de la finca raíz tras el pinchazo de la burbuja de precios entre 2007 y lo corrido de 2009 (Altman, 2009).

En un estudio para Colombia, Clavijo, et al. (2004) enfatizan que los ciclos hipotecarios en el país son pronunciados, con valorizaciones de hasta $50-100 \%$ real en las fases de auge y con desvalorizaciones del $20-30 \%$ real, los cuales pueden durar entre 4-6 años al alza con caídas rápidas en 1-2 años.

Estos ciclos pueden generar efectos encadenados muy perjudiciales para la economía. Según Case (2000), el sector de la construcción juega un rol muy importante en la amplificación y la propagación de los choques en la economía. Bernanke et al. (1999), al igual que Case, argumentan que existen razones teóricas y empíricas para creer que, cambios en el precio de la finca raíz generan incrementos y reducciones en la demanda por inversión y consumo. Si las instituciones financieras utilizan propiedades como garantía para préstamos, entonces un declive en su precio reducirá el valor de la garantía, y con ello la disponibilidad de fondos para prestar.

Adicionalmente, un declive en el precio de la finca raíz y de las hipotecas puede agotar el capital bancario debido a que un porcentaje muy importante del portafolio de los bancos se encuentra en hipotecas. El agotamiento del capital financiero por

parte de los bancos trae como consecuencia una disminución en la oferta de crédito que perjudica la inversión. Los factores anteriormente mencionados hacen que la evolución de los precios de la finca raíz amplifiquen y propaguen los choques en la economía. 


\section{3. ¿Cómo afectó la crisis económica mundial al sector edificador en Colombia?}

El sector constructor colombiano mostró claras señales de desaceleración a partir de 2008 asociada con factores internos, principalmente con el fin de su ciclo expansivo (Mesa, Restrepo y Aguirre, 2008). En efecto, la Gráfica 2 ilustra que el sector presentó un periodo de auge entre finales de 2002 hasta el año 2007.

Gráfica 2

Ciclo del sector edificador colombiano

Hodrick-Prescott Filter

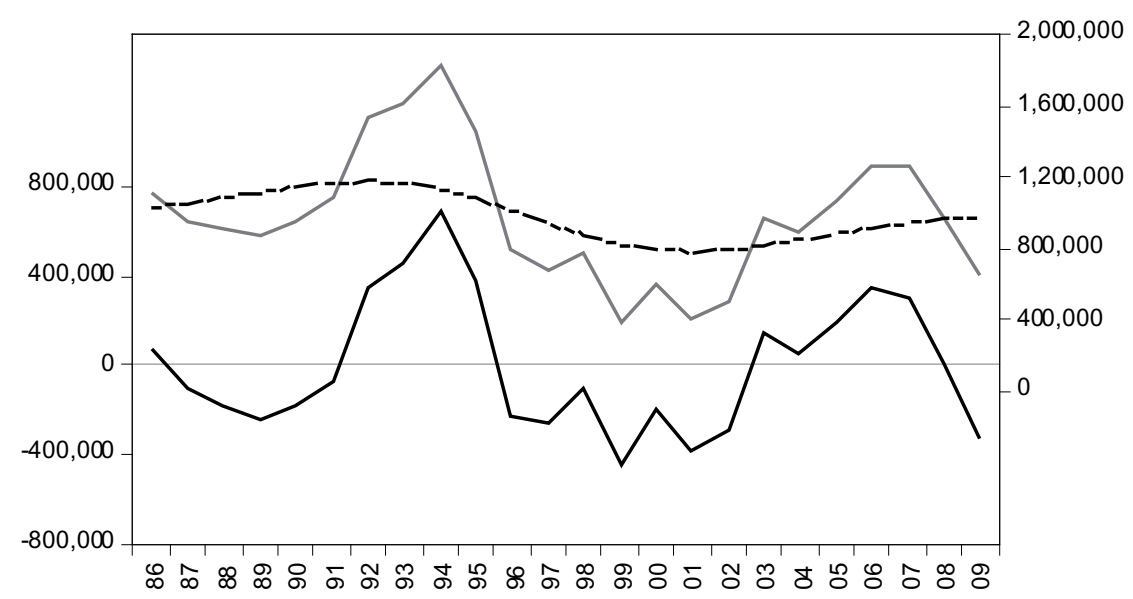

- Licencias de construcción --- Tendencia _ Ciclo

Fuente: elaboración de los autores con base en datos del DANE.

Si bien el renglón edificador registró signos de desaceleración que obedecen a su ciclo económico normal, no son menos ciertos los efectos negativos que tuvo la crisis económica global sobre los determinantes de la actividad edificadora. Esta situación se hizo evidente para el cuarto trimestre de 2008 y el primer trimestre de 2009, en especial en el caso de la construcción de vivienda. En la Gráfica 3 se observa la sensibilidad del sector construcción a las condiciones de la crisis económica global. 
Es así como las licencias de construcción para el cuarto trimestre de 2008, respecto al cuarto trimestre del año 2007, presentó una disminución de 26.4\%. Entre tanto, las solicitudes de licencias descendieron en un 30.2\% para el primer trimestre de 2009 con respecto a igual periodo de 2008.

\section{Gráfica 3}

Licencias de construcción para vivienda en Colombia. Área (metros cuadrados)

Enero de 2000 a Marzo de 2010

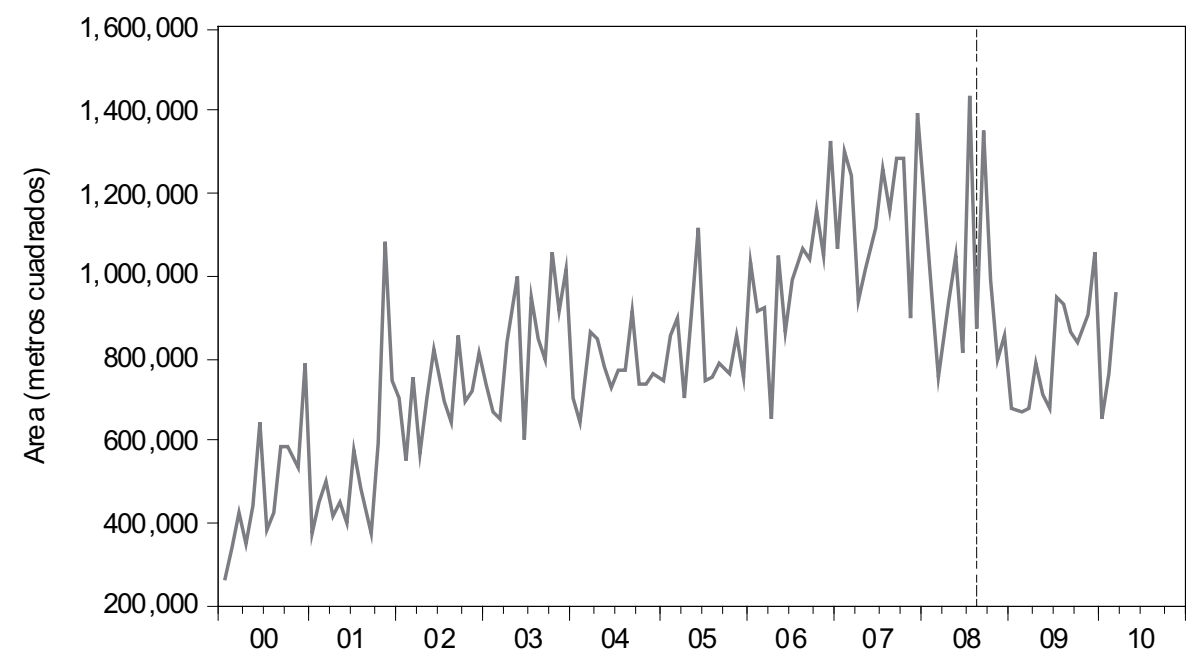

Fuente: gráfico de los autores con base en los datos de DANE.

En la literatura sobre contagio y crisis financiera se ha detectado que los canales de contagio más comunes son los vínculos a través del comercio internacional; los préstamos desde los centros financieros mundiales hacia los mercados emergentes; las variaciones en los precios de las materias primas y fenómenos de carácter psicológico. Neal y Weidenmier (2002), Stiglitz (2010), Bordo y Murshid (2000)² y Glick y Rose (1998) advierten que, incluso la transmisión de los choques externos

2 En su último libro Roubini y Mihm (2010) advierten de las consecuencias de las políticas anticíclicas adoptadas durante la crisis económica global de 2008-2009 sobre el futuro de las finanzas públicas en la mayoría de los países. 
negativos por la vía de los canales comercial y financiero pueden ocurrir de manera súbita cuando el grado de globalización del país es alto y se encuentra estrechamente vinculado a otras economías previamente afectadas. ${ }^{3}$

En Colombia, en el transcurso de los últimos veinte años numerosos analistas han desarrollado una extensa literatura económica estableciendo las principales variables, tanto internas como externas, que influyen en el comportamiento del sector edificador. Entre ellos se encuentran los trabajos publicados por Junguito et al. (1995), Cárdenas y Bernal (1996), Clavijo et al. (2005), Cárdenas y Hernández (2006) y Camacol (2008).

Los autores señalan que, al analizar en conjunto el mercado hipotecario en Colombia y su relación con los determinantes a nivel nacional e internacional, se debe tener en cuenta un enfoque de demanda y de oferta de finca raíz. En este orden de ideas, los factores que inciden positivamente tanto en la oferta como en la demanda de vivienda nueva son el PIB real, la disponibilidad del crédito y las remesas. Por su parte, aquellas variables que afectan negativamente al sector son la tasa de interés real hipotecaria, la tasa de desempleo y los costos de la construcción de vivienda.

Así pues, la crisis financiera y económica global terminó por afectar el comportamiento de la economía y el sector de la construcción en Colombia a través de los canales comercial y financiero, los cuales se relacionan con la reducción en el ingreso disponible de los hogares, las remesas, las tasas de interés, la liquidez y el crédito y las variaciones en los precios de los insumos de la construcción (Ocampo, 2009).

En este sentido, entonces vale la pena analizar el comportamiento de los determinantes de la actividad constructora durante la crisis económica internacional, con el fin de establecer los efectos que tuvo la recesión global sobre el segmento constructor.

En cuanto al canal comercial, la reducción de los ingresos en las principales economías mundiales afecta el crecimiento y el nivel de empleo de Colombia por la vía de una reducción de la cantidad demanda y del precio de sus exportaciones, lo cual afectó el nivel de ingreso disponible de los hogares, tal y como se aprecia en la Gráfico 4.

Algunas instituciones y autores han realizado estimaciones sobre el impacto y costo de la crisis económica global para los países en desarrollo. El Banco Mundial predice que la crisis aumentará el número de personas que viven en pobreza extrema y obstaculizará el logro de los objetivos de desarrollo del milenio (Banco Mundial, 2010). Otras estimaciones nada alentadoras son las de FMI (2009), Action Aid (2009), Oxfam (2009) y Devarajan (2009). 


\section{Gráfica 4}

\section{Producto Interno Bruto}

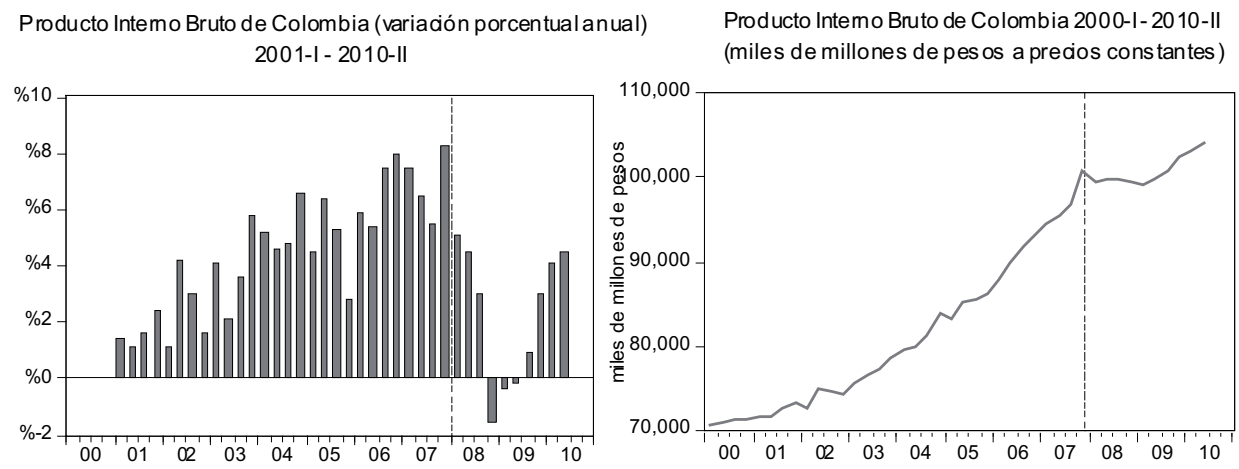

Fuente: gráfico de los autores con base en los datos de DANE.

El ingreso disponible de los hogares es tal vez uno de los factores más importantes relacionados a la demanda de finca raíz. La recesión en las principales economías desarrolladas, así como la desaceleración del crecimiento en Colombia, influyó en la disminución de la inversión de los hogares. ${ }^{4}$

Adicionalmente, la crisis global afecta el ingreso disponible de los emigrantes colombianos, hecho que impacta negativamente en la entrada de remesas al país, las cuales cayeron $-11.6 \%$ en el último trimestre de 2008, justo en los momentos de mayor incertidumbre de la crisis mundial (ver Gráfica 5). Según el Fondo Multilateral de Inversiones (2009), en 2009 Colombia fue el tercer mayor receptor de remesas en América Latina, sólo superado por México y Brasil.

En la Gráfica 5 se puede apreciar el sostenido aumento de los ingresos por remesas en Colombia, al igual que su desaceleración y caída desde el último trimestre de 2008 hasta el tercer trimestre de 2009. ${ }^{5}$ Es probable que parte de los ingresos de remesas sean utilizados por los hogares colombianos para el pago parcial de cánones de arrendamientos, cuotas de financiación de sus viviendas o cuotas iniciales para

4 Estudios interesantes realizados para Colombia son los de Diaz et al. (1993), que explican de manera detallada la interrelación que existe entre el PIB total de la economía y la actividad de la construcción; Junguito et al. (1995) concluyen que el crecimiento del producto interno bruto es uno de los principales factores que afectan la demanda por edificaciones; y, Herrera (1998 ) explica el efecto en el comportamiento de las licencias de construcción ante variaciones del PIB per capita, encontrando una alta elasticidad entre estas variables.

5 Aunque el valor de las remesas suele presentar variaciones de carácter estacional, una vez eliminado este efecto, se encontró que la caída en el valor de las remesas en Colombia durante el año 2009 fue el resultado del impacto de la crisis mundial en los ingresos de los colombianos en el exterior y no un fenómeno netamente estacional. 
la compra de las mismas. Un deterioro en el crecimiento de este indicador asociado al ingreso disponible de los hogares colombianos afecta la capacidad de pago de los mismos y termina por influir en los indicadores del sector de la construcción.

\section{Gráfica 5}

Ingresos por remesas en Colombia. Millones de dólares

Enero de 2000 a Junio de 2010

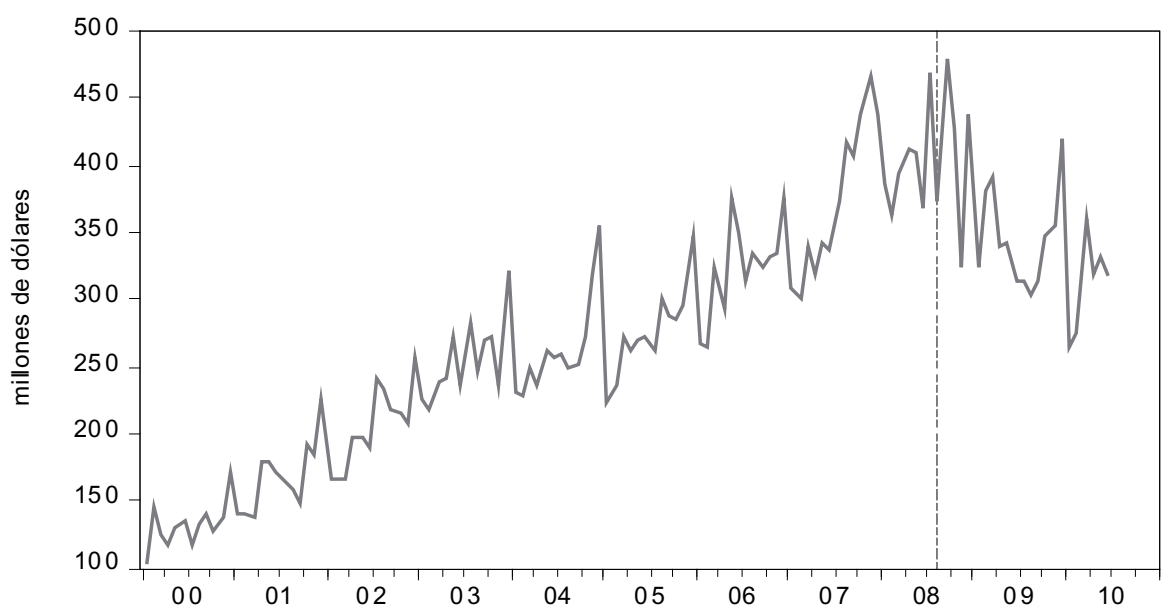

Fuente: gráfico de los autores con base en los datos del Banco de la República.

Según Garay (2006), parte de los ingresos provenientes de las remesas va destinada a la compra o construcción de vivienda. En Colombia, la aprobación de licencias de construcción (vivienda y otros usos) disminuyó $9.9 \%$ en 2008 y $23 \%$ en 2009; adicionalmente, en 2008 el crecimiento de las remesas fue de $7.8 \%$, apenas la mitad de lo que crecieron en 2007, en tanto que en el 2009 la situación con las remesas fue incluso peor, cayendo $16.8 \%$ frente al año anterior.

Debido al fenómeno conocido como fly to quality, en el cual los inversionistas buscaron refugio en activos financieros más seguros, en Colombia se experimentó un fuerte proceso de depreciación del peso frente al dólar entre julio de 2008 y febrero de 2009. ${ }^{6}$ Este proceso de depreciación contribuyó, en efecto, como señala Ocampo

6 El término fly to quality se refiere al desplazamiento de los capitales especulativos desde los activos financieros de mayor riesgo a los más seguros (dólares, oro o Bonos del Tesoro de los Estados Unidos), como reacción a la aparición de situaciones de riesgo o incertidumbre en el contexto económico. 
(2009), a compensar la caída en el ingreso disponible de los hogares que reciben remesas. En tal sentido, Ocampo considera que el efecto de la disminución de las remesas es el canal de contagio menos relevante.

Como se ha mencionado a lo largo del documento, la contracción de la economía mundial terminó por afectar el aparato productivo nacional y, en consecuencia, se deterioró el mercado laboral del país. Así pues, se evidenció durante la crisis global una disminución de los ingresos laborales de las familias que obedeció en gran parte al incremento de la tasa de desempleo. La Gráfica 6 advierte a partir del año 2008 el cambio de tendencia que presentó la tasa de desempleo y su marcado incremento para inicios de 2009. Esta disminución de ingresos hace menos posible la adquisición de vivienda nueva.

\section{Gráfica 6}

Tasa de desempleo en Colombia (13 ciudades)

Enero 2000 a Septiembre 2010

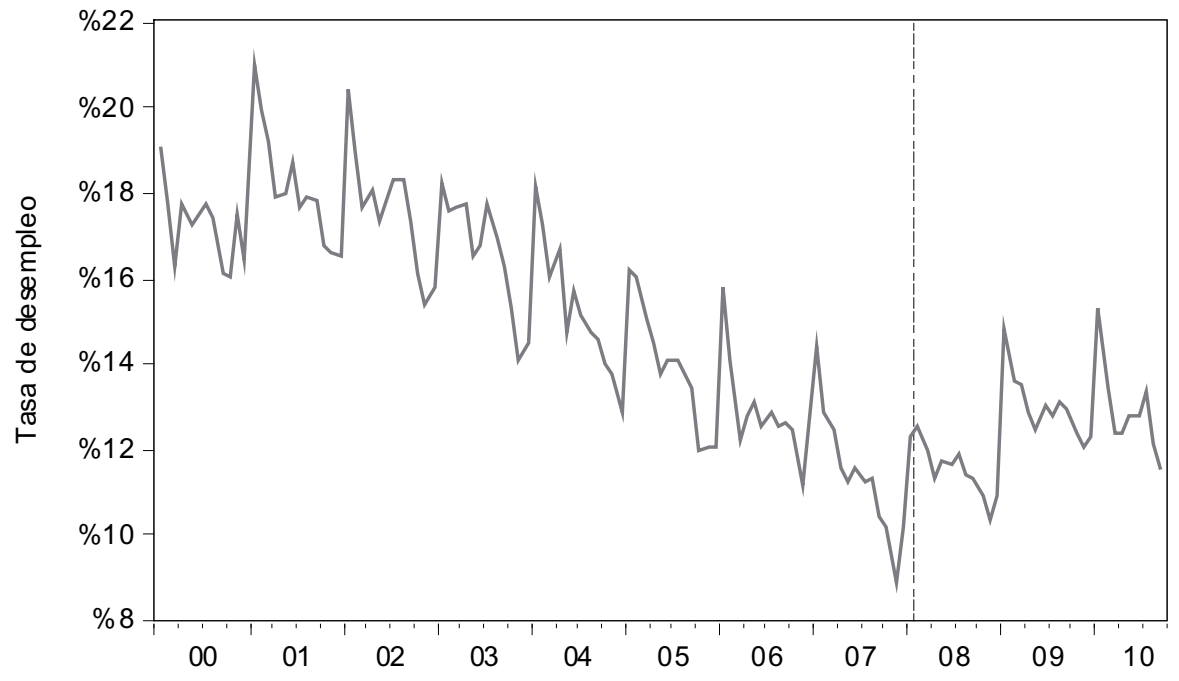

Fuente: gráfico de los autores con base en los datos del Banco de la República.

En el escenario de desaceleración de la economía colombiana de finales de 2008, y como respuesta a la crisis económica internacional, el Banco de la República identificó un claro margen de reducción de los tipos de interés (ver Gráfica 7). Los ni- 
veles de la tasa de interés influyen tanto en las decisiones de los constructores para invertir -lado de la oferta- como en las decisiones de los compradores -lado de la demanda.

\section{Gráfica 7}

Tasa de intervención de política monetaria

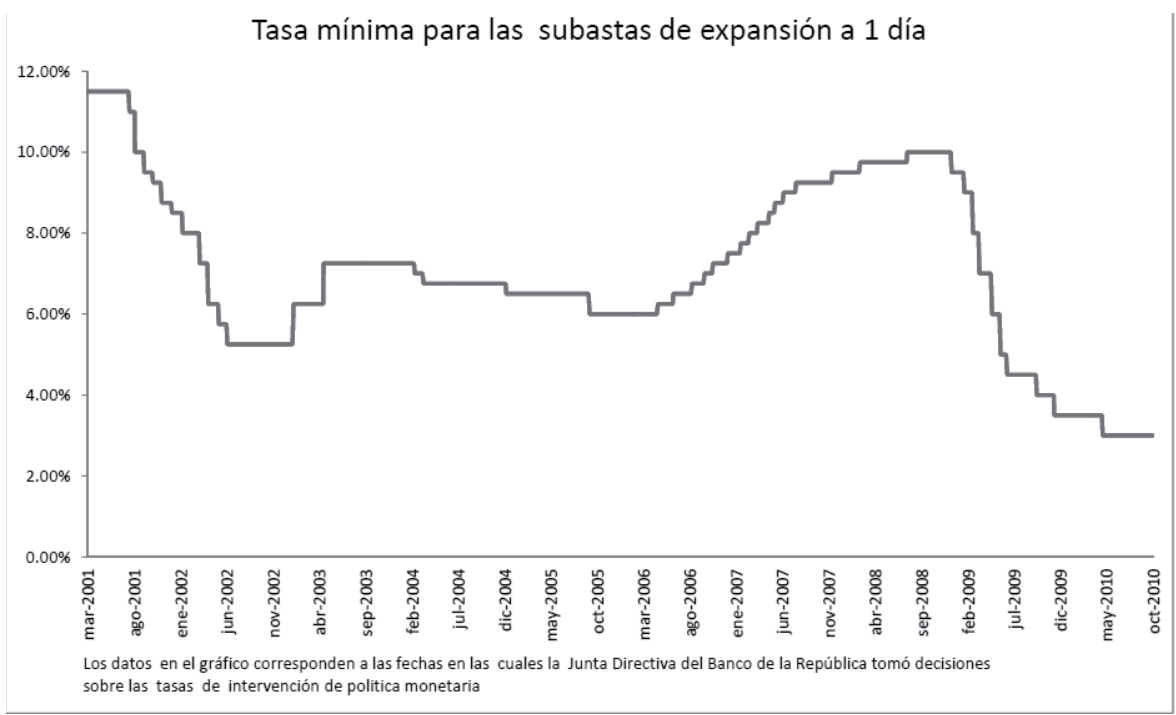

Fuente: Banco de la República.

La sensibilidad del sector edificador a la tasa de interés obedece, por un lado, a que el costo de la mayor parte de los proyectos inmobiliarios es demasiado alto para ser sorteado por un solo inversionista o incluso por un grupo de ellos, por lo que éstos suelen verse obligados a financiar sus proyectos con el sistema financiero. Las altas tasas de interés harán que muchos de estos proyectos parezcan inviables, mientras que las menores tasas de interés inducirán a mayores solicitudes de créditos (Keynes, 1936). Por otro lado, En el caso de los compradores, el costo total del bien inmueble, incluido el costo de financiación, será menor cuanto menores sean las tasas de interés, aumentando la demanda de finca raíz e incentivando la construcción de nuevos proyectos. En este sentido, entonces, la política monetaria de orden expansivo del Banco de la República ejerció un impacto positivo en el renglón edificador colombiano. 
Para Berger y Ellis (2004), el sector de la construcción es considerado el sector económico más sensible a las variaciones de la tasa de interés. Camacol (2008), por su parte, encontró para Colombia que, un aumento de un punto porcentual en la tasa de interés hipotecaria reduce en $1.3 \%$ las licencias de construcción.

En cuanto a la oferta de finca raíz, la reducción en los precios internacionales de los materiales básicos de construcción, como cemento y acero, ha contribuido a la caída de los costos de la construcción en Colombia, como se puede ver en el Cuadro 1 donde se muestra que, desde septiembre de 2008 hasta julio de 2009 ha predominado un comportamiento deflacionario en los costos. Sin embargo, los constructores nacionales no alcanzaron a beneficiarse del todo debido a la fuerte devaluación del peso ocurrida entre julio de 2008 y febrero de 2009; dicha devaluación, entonces, compensó buena parte los beneficios de la reducción de las materias primas.

Cuadro 1

Colombia: Índice de Costos de la Construcción de Vivienda (ICCV)

Enero 2001-Octubre 2010

\begin{tabular}{|l|r|r|r|r|r|r|r|r|r|r|r|}
\hline \multicolumn{1}{|c|}{ Mes } & $\mathbf{2 0 0 1}$ & $\mathbf{2 0 0 2}$ & $\mathbf{2 0 0 3}$ & $\mathbf{2 0 0 4}$ & $\mathbf{2 0 0 5}$ & $\mathbf{2 0 0 6}$ & $\mathbf{2 0 0 7}$ & $\mathbf{2 0 0 8}$ & $\mathbf{2 0 0 9}$ & $\mathbf{2 0 1 0}$ \\
\hline Enero & 2,67 & 1,07 & 1,84 & 1,65 & 0,80 & 1,52 & 0,71 & 1,42 & 0,52 & 0,58 \\
\hline Febrero & 1,58 & 1,44 & 2,28 & 2,44 & 0,59 & 0,51 & 0,99 & 1,56 & 0,44 & 0,35 \\
\hline Marzo & 0,57 & 0,39 & 1,08 & 2,24 & 0,46 & 0,57 & 0,80 & 0,75 & $-0,02$ & 0,46 \\
\hline Abril & 0,48 & 0,29 & 0,51 & 0,82 & 0,39 & 0,54 & 0,48 & 0,50 & $-0,18$ & 0,44 \\
\hline Mayo & 0,29 & 0,38 & 0,69 & 0,50 & 0,45 & 0,69 & 0,17 & 0,32 & $-0,21$ & 0,53 \\
\hline Junio & 0,37 & 0,15 & 0,15 & 0,17 & 0,20 & 0,76 & $-0,03$ & 0,91 & $-0,37$ & 0,25 \\
\hline Julio & 0,98 & 0,18 & 0,15 & 0,25 & $-0,26$ & 1,13 & $-0,10$ & 0,32 & $-0,02$ & $-0,07$ \\
\hline Agosto & 0,00 & 0,24 & 0,25 & 0,11 & 0,06 & 0,46 & 0,22 & 0,20 & $-0,29$ & $-0,34$ \\
\hline Septiembre & 0,11 & 0,30 & 0,21 & $-0,06$ & $-0,23$ & 0,32 & 0,16 & $-0,01$ & 0,05 & $-0,33$ \\
\hline Octubre & 0,45 & 0,65 & 0,27 & $-0,17$ & $-0,03$ & 0,20 & 0,30 & $-0,29$ & $-0,21$ & $-0,12$ \\
\hline Noviembre & 0,23 & 0,67 & 0,6 & $-0,09$ & 0,11 & $-0,11$ & 0,13 & $-0,31$ & $-0,56$ & \\
\hline Diciembre & 0,24 & 0,66 & 0,38 & $-0,21$ & 0,12 & $-0,12$ & 0,33 & $-0,19$ & $-0,28$ & \\
\hline En año corrido & $\mathbf{8 , 2 5}$ & $\mathbf{6 , 5 9}$ & $\mathbf{8 , 7 2}$ & $\mathbf{7 , 8 8}$ & $\mathbf{2 , 6 9}$ & $\mathbf{6 , 6 4}$ & $\mathbf{4 , 2 3}$ & $\mathbf{5 , 2 9}$ & $\mathbf{- 1 , 1 2}$ & \\
\hline
\end{tabular}

Fuente: DANE.

Por su parte, y contrario a la fuerte contracción que experimentó el establecimiento financiero para finales de los noventa, en el curso de la presente década las instituciones financieras colombianas recuperaron la solidez, lo que les permitió te- 
ner disponibilidad de recursos y no cerrar el canal de crédito durante la crisis internacional. De acuerdo con Avendaño (2009) el canal del crédito en Colombia sigue funcionando y no se han detectado problemas con las financiaciones.

En la Gráfica 8 se aprecian los desembolsos totales para todas las modalidades de crédito y los desembolsos adjudicados para crédito de vivienda, desde el año 2004 hasta 2010. Se advierte un quiebre entre mediados de 2008 y principios de 2009 producto, en gran medida, de la desconfianza en la economía tras el estallido de la crisis y no por la falta de recursos financieros, pues los desembolsos para finales de 2009 retomaron su tendencia creciente. Esta situación impactó positivamente el renglón edificador ya que el crédito financiero es una de las fuentes primordiales para la construcción y compra de vivienda.

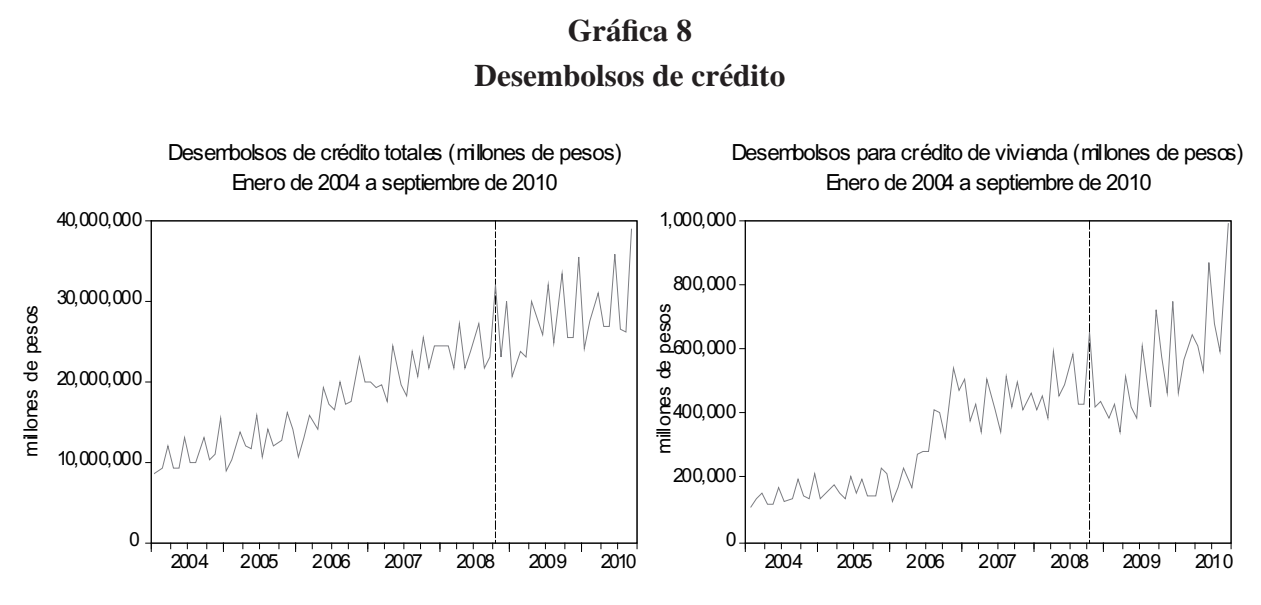

Fuente: gráficos de los autores con base en los datos de la Superintendencia Financiera.

Como respuesta a la crisis financiera y económica global se implementaron varias políticas expansivas para contrarrestar los efectos e impactos sobre la economía nacional que, en últimas, influyeron en variables claves del sector constructor.

Por una parte, la disminución en la tasa de intervención del Banco de la República hizo menos costoso el crédito para los oferentes (constructores) y demandantes (hogares) de vivienda nueva. Por otro lado, la política de vivienda introducida por el gobierno nacional, que consistió en subsidiar la tasa de interés hipotecaria, impulsó fuertemente el sector. 
Así pues, estas estrategias de políticas expansivas tanto fiscal como monetaria han contribuido a compensar los efectos negativos sobre el sector y contribuir a una pronta recuperación del mismo (Revista Dinero, 2009).

\section{Conclusiones}

Las causas de la crisis económica global desatada en los Estados Unidos estuvieron estrechamente ligadas al desempeño del sector de la construcción y sus vínculos con el sector financiero. Primero con el surgimiento de una burbuja en los precios inmobiliarios y, posteriormente con su explosión y el inicio del proceso deflacionario que se extendió a las bolsas de valores y afectó los balances del sistema financiero y se tradujo finalmente en la peor recesión de la economía americana desde la gran depresión.

La crisis en el sector construcción se agravó cuando el endeudamiento se elevó y, unido a la caída de los precios de la vivienda, terminó por hacer que el valor de los créditos fuera mayor que el valor de los bienes inmuebles que los garantizan, lo cual generó incentivos para la suspensión de pagos de las cuotas y creó las condiciones para el inicio de una crisis financiera que se extendió al sector real.

En un mundo altamente globalizado en sus intercambios financieros y comerciales, los efectos de la crisis americana se hicieron sentir rápidamente en todos los rincones de la tierra, incluida Colombia.

Uno de los sectores nacionales afectados por la crisis ha sido el sector edificador, el cual, por su importancia como generador de empleo y encadenamientos productivos, resulta muy sensible para el resto de la economía, en especial porque su derrumbe podría generar costos sociales difíciles de reparar en el corto y mediano plazo.

Así pues, la crisis financiera y económica global terminó por afectar el comportamiento del sector de la construcción en Colombia a través de los canales comercial

y financiero, los cuales se relacionan con la reducción en el ingreso disponible de los hogares, las remesas, las tasas de interés, la liquidez y el crédito y las variaciones en los precios de los insumos de la construcción.

Específicamente, los canales de transmisión de la crisis global al sector edificador colombiano están asociados con la desconfianza económica, la caída del PIB, el aumento en la tasa de desempleo así como también con la caída de las remesas, variables que afectaron negativamente la demanda de edificaciones en Colombia. 
No obstante, la disminución en la tasa de intervención del Banco de la República hizo menos costoso el crédito para los oferentes (constructores) y demandantes (hogares) de vivienda nueva. En la misma línea, la política de vivienda introducida por el gobierno nacional, que consistió en subsidiar la tasa de interés hipotecaria, impulsó fuertemente el sector.

Otros efectos están relacionados con el precio de las materias primas. La recesión global ha contribuido a la reducción de los precios de los bienes básicos a nivel global, favoreciendo la reducción de los costos de los materiales de construcción y generando un incentivo adicional para los constructores.

Teniendo en cuenta el funcionamiento de los canales de contagio analizados en este artículo, es importante resaltar que para mantener un sector de la construcción dinámico en los años que sobrevienen a la crisis, es recomendable que la política económica garantice la estabilidad o aumento de los ingresos disponibles de los hogares, el poder adquisitivo de las remesas provenientes del exterior, un nivel bajo de tasas de interés y, mediante el control de la inflación y las fluctuaciones de los tipos de cambio, un nivel estable de precios de los insumos de la construcción. Asimismo, sería deseable también mantener e incluso ampliar las políticas de subsidios o estímulos a la compra de vivienda nueva.

Finalmente, se espera que este artículo contribuya como insumo para la producción de nuevos proyectos de investigación y artículos especializados que profundicen en el conocimiento sobre el impacto de los distintos canales de contagio de las crisis externas en el sector de la construcción para el caso colombiano.

\section{Bibliografía}

Action Aid (2009), "Where does it hurt? The impact of the international crisis on developing Countries", <http://www.actionaid.org.uk/doc_lib/where_does_it_ hurt_nal.pdf $>$.

Altman, R. (2009), “The Great Crash, 2008: A Geopolitical Setback for the West”, Foreign Affairs, vol. 88, núm. 1.

Avendaño, H. (2009), "De la crisis financiera a la crisis económica mundial", Vox Populi, núm. 8, pp. 1-30.

Banco de la República (2009), "Evolución de los ingresos por remesas de trabajadores en 2008”, Comunicado de prensa, $<$ http://www.banrep.gov.co/sala-prensa/ index.html\#5022009>. 
¿Cómo afectó la crisis económica global al sector edificador en Colombia?

Un análisis de los canales de contagio

Banco Mundial (2010), "Global Monitoring Report 2009: The MDGs after the Crisis”, <http://extop-workflow.worldbank.org/extop/ecommerce/catalog/ product?context $=$ drilldown\&item_id $=9659438>$.

Berger-Thompson, L. y L. Ellis (2004), "Housing Construction Cycles and Interest Rates, Economic Group Reserve Bank of Australia”, <http://ideas.repec.org/p/ rba/rbardp/rdp2004-08.html>.

Bernanke, B., M. Gertler y S. Gilchrist (1999), "The financial accelerator in a quantitative bussines cycle framework", NBER Working Paper, núm. 6455, National Bureau of Economic Research, Inc.

Bordo, M. y A. Murshid (2000), “Are financial Crises Becoming Increasingly More Contagious? What is the Historical Evidence on Contagion?", NBER Working Paper, núm. 7900, National Bureau of Economic Research, Inc.

CAMACOL (2009), Informe Económico No. 17, <http://www.camacol.org.co/adminSite/Archivos/EE_Coy20090707115723.pdf $>$.

CAMACOL (2008), “Actividad edificadora en Bogotá y Cundinamarca”, Departamento de Estudios Económicos, Bogotá, <http://www.camacol.org.co/adminSite/Archivos/ArtPres_20080414025426.pdf >.

Case, K.E. (2000), "Real estate and the macroeconomy", Brookling Papers on Economic Activity, vol. 2, pp. 119-145.

Cárdenas, M. (2006-a), "El sector de materiales de construcción en Bogotá-Cundinamarca", Fedesarrollo, Bogotá, <http://www.fedesarrollo.org/contenido/articulo.asp? chapter $=192 \&$ article $=406>$.

Cárdenas, M. (2006-b), "El sector de la construcción en Colombia", FedesarrolloCamacol, Bogotá, <http://www.cardenasmauricio.com/ingles/images/presentaciones/camacol.pdf $>$.

Cárdenas, M. y R. Bernal (1997), “Auge y crisis de la construcción en Colombia: causas y consecuencias”, Revista Camacol, vol. 1, núm. 1, pp. 8-32.

Chenery, H. y T. Watanabe (1958), "International comparisons of the structure of production”, Econometrica, vol. 26, núm. 4.

Clavijo, S., M. Janna y S. Muñoz (2004), "La vivienda en Colombia: sus determinantes socioeconómicos y financieros", Documento de trabajo Núm. 300, Banco de la República, Bogotá.

DANE-a (2009), Producto Interno Bruto, Cuarto trimestre de 2008, Boletín de Prensa, Bogotá, <http://www.dane.gov.co/files/investigaciones/boletines/pib/bolet_PIB_IVtrim08.pdf $>$. 
DANE-b (2009), Indicadores Económicos alrededor de la Construcción, Tercer trimestre de 2009, Boletín de Prensa, Bogotá, <http://www.dane.gov.co/files/investigaciones/boletines/pib_const/Bol_ieac_IIItrim09.pdf $>$.

DANE-c (2010), Principales indicadores del mercado laboral marzo de 2010, Boletín de Prensa, Bogotá, <http://www.dane.gov.co/files/investigaciones/boletines/ech/ech/bol_ech_mar10.pdf $>$.

Devarajan, S. (2009), “Africa: Least integrated but worst hit by the crisis", $<$ http:// africacan.worldbank.org/africa-least-integrated-but-worst-hit-by-the-crisis>.

Diaz, J., F. Gaitan, G. Piraquive y M. Ramirez (1993), “Dinámica de la construcción entre 1950 y 1991”, Planeación y Desarrollo, vol. 25, Bogotá.

Eichengreen, B. y M. Bordo (2002), "Crises Now and Then: What Lessons from the Last Era of Financial Globalization", NBER Working Paper, núm. W8716, National Bureau of Economic Research, Inc.

Fondo Multilateral de Inversiones, MIF (2009), "Remesas a América Latina y el Caribe 2009 (millones de dólares)", <http://www.iadb.org/mif/remesas_map. cfm?language $=$ Spanish $>$.

Ferguson, N. (2008), The Ascent of Money: A financial History of the World, The Penguin Press, New York.

Garay, L. (2006), "El colectivo colombiano residente en la comunidad de Madrid (España): caracterización socio-económica, inserción laboral e integración social", en Colombia nos une. Memorias II Seminario sobre migración internacional colombiana y la conformación de comunidades transnacionales, Fondo Editorial Ministerio de Relaciones Exteriores, Bogotá.

Glick, R. y A. Rose (1998), "Contagion and trade: Why are currency crises regional?", NBER Working Paper, núm. 6806, National Bureau of Economic Research, Inc.

Herrera, F. (1988), "Notas sobre algunos aspectos del mercado de la edificación en Colombia", Revista Camacol, núm. 41, Bogotá.

Hirschman, A.O. (1958), The Strategy of Economic Development, New Haven: Yale University Press, edición en español: La estrategia de desarrollo económico, México, 1961, FCE.

International Monetray Fund (2009), "The importance of the global international crisis for low income countries", <http://www.imf.org/external/pubs/ft/ books/2009/global.n/global.n.pdf>. 
¿Cómo afectó la crisis económica global al sector edificador en Colombia?

Un análisis de los canales de contagio

Junguito, R., E. López, M. Misas y E. Sarmiento (1995), "La edificación y la política económica", Borradores de Economía, núm. 41, Banco de la República, pp. 1-27.

Kaminsky, G. y C. Reinhart (1999), "The causes of banking and balance-of-payments problems", The American Economic Review, vol. 89, núm. 3, pp. 473-500.

Keynes, J. (1936), Teoría general sobre el empleo, el interés y el dinero, Fondo de Cultura Económica, edición del año 2000, México.

Kindleberger, C., R. Aliber y R. Solow (2001), Manias, Panics and Crashes: A History of Financial Crises, McMillan Press, London.

Krugman, P. (2009), De vuelta a la economía de la Gran Depresión y la crisis del 2008, Editorial Norma, Bogotá.

Mesa, R., D. Restrepo y Y. Aguirre (2008), "Crisis externa y desaceleración de la economía colombiana en 2008-2009”, Perfil de Coyuntura Económica, núm. 12, pp. 31-67.

MIF-Interamerican Development Bank (2009), "Remittances to Latin America and the Caribbean 2008 (US\$ millions)", <http://www.iadb.org/mif/remesas_map. cfm?language $=$ English \&parid $=5>$.

Miller, H. (1924), "Theories of crises and cycles in the Unites States", The quarterly Journal of Economics, 2(29), pp. 294-329.

Neal, L. y M. Weidenmier (2002), "Crisis in the global economy from tulips to today: contagion and consequences", NBER Working Papers, núm. 9147, National Bureau of Economic Research, Inc.

Ocampo, J. (2009), "Impactos de la crisis mundial sobre América Latina", Revista CEPAL, núm. 97, pp. 9-32.

Oxfam (2009), "Initial Assessment of impact of Global Economic Crisis in Developing Countries", <http://www.oxfam.org.uk/applications/blogs/presso $\varnothing$ ce/?p=4011>.

Rajan, R. y L. Zingales (2004), Saving Capitalism from the Capitalists: Unleashing the Power of Financial Markets to Create Wealth and Spread Opportunity, Princeton University Press, New Jersey.

Rasmussen, P. N. (1956), Studies in intersectoral relations, Einar Harcks Forlag \& North-Holland Publishing Company, Copenhague and Amsterdam.

Reid, M. (2008), "Nov 11. The party's over: A Special Report on Spain”, The Economist, vol. 389, núm. 8605, pp.3-20.

Reinhart, C. y K. Rogoff (2008), "This time is different: a panoramic view of eight centuries of financial crises", NBER Working Paper, núm. 13882, National Bureau of Economic Research, Inc., pp.1-62. 
Revista Dinero (2009), "Le llegó el turno a América Latina”, núm. 317, p. 39., ISSN 0122-1531.

Revista Dinero (2009), “Vivienda: ¡Aproveche los beneficios!”, núm. 324, pp. 8490, <http://www.dinero.com/noticias-dinero-y-usted/vivienda-aproveche-beneficios/59280.aspx>.

Roubini, N. y S. Mihm (2010), Crisis Economics: A Crash Course in the Future of Finance, Penguin Books.

Shiller, J. (2005), “Irrational Exuberance”, Segunda edición, Princeton University Press.

Soros, G. (2008), El nuevo paradigma de los mercados financieras, Editorial Tauros. Bogotá.

Stiglitz, J. (2010), Caida libre: Estados Unidos, el libre mercado y el hundimiento de la economía mundial, Editorial Taurus, Barcelona.

The Economist Intelligence Unit (2010), <http://viewswire.eiu.com/index. asp?layout=VWCountryVW3\&region_id=\&country_id=1510000151 >.

Toffler, A., H. Toffler, et al. (2009), La crisis Económica Mundial. La caída del dinero por qué el mundo está haciendo las preguntas equivocadas, Editorial Oveja Negra, Bogotá. 